\title{
Ultrafast Generation of Fundamental and Multiple-Order Phonon Excitations in Highly Enriched $(6,5)$ Single-Wall Carbon Nanotubes
}

Yong-Sik Lim, ${ }^{\dagger}$ Ahmad R. T. Nugraha, ${ }^{\ddagger}$ Sung-Jae Cho, ${ }^{\dagger}$ Min-Young Noh, ${ }^{\dagger}$ Eun-Jin Yoon, ${ }^{\S}$ Huaping Liu,

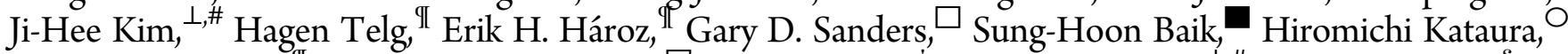
Stephen K. Doorn, ${ }^{\text {,I }}$ Christopher J. Stanton, ${ }^{\square}$ Riichiro Saito, ${ }^{\ddagger}$ Junichiro Kono, ${ }^{*, \perp, \#}$ and Taiha Joo ${ }^{*, \S}$

${ }^{\dagger}$ Department of Nano Science and Mechanical Engineering and Nanotechnology Research Center, Konkuk University, Chungju, Chungbuk 380-701, Republic of Korea

${ }^{\ddagger}$ Department of Physics, Tohoku University, Sendai 980-8578, Japan

${ }^{\S}$ Department of Chemistry, POSTECH, Pohang 790-784, Republic of Korea

"Beijing National Laboratory for Condensed Matter Physics, Institute of Physics, Chinese Academy of Sciences, Beijing 100190, China

${ }^{\perp}$ Department of Electrical and Computer Engineering and ${ }^{\#}$ Department of Physics and Astronomy, Rice University, Houston, Texas 77005, United States

${ }^{\text {II }}$ Center for Integrated Nanotechnologies, Los Alamos National Laboratory, Los Alamos, New Mexico 87545, United States

$\square$ Department of Physics, University of Florida, Gainesville, Florida 32611, United States

Quantum Optics Research Division, Korea Atomic Energy Research Institute, Daejeon 305-353, Republic of Korea

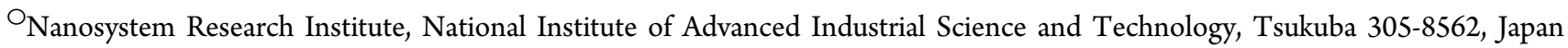
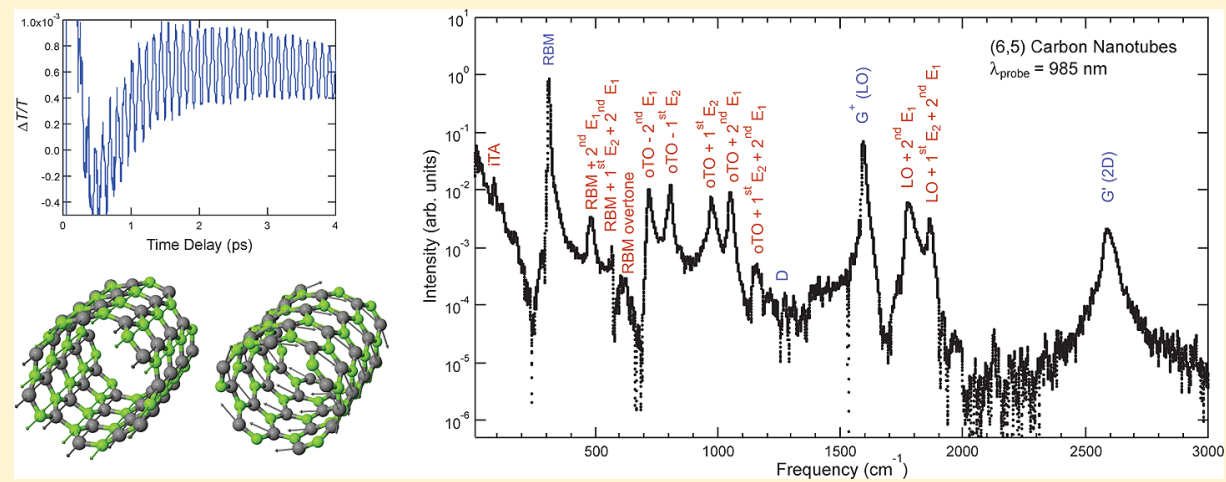

ABSTRACT: Using a macroscopic ensemble of highly enriched $(6,5)$ single-wall carbon nanotubes, combined with high signalto-noise ratio and time-dependent differential transmission spectroscopy, we have generated vibrational modes in an ultrawide spectral range $\left(10-3000 \mathrm{~cm}^{-1}\right)$. A total of 14 modes were clearly resolved and identified, including fundamental modes of $A, E_{1}$, and $\mathrm{E}_{2}$ symmetries and their combinational modes involving two and three phonons. Through comparison with continuous wave Raman spectra as well as calculations based on an extended tight-binding model, we were able to identify all the observed peaks and determine the frequencies of the individual and combined modes. We provide a full summary of phonon frequencies for $(6,5)$ nanotubes that can serve as a basic reference with which to refine our understanding of nanotube phonon spectra as well as a testbed for new theoretical models.

KEYWORDS: Single-wall carbon nanotubes, coherent phonons, single chirality, resonance Raman spectroscopy

$\mathrm{R}$ ecent advances in separation and sorting techniques have allowed researchers to prepare highly enriched, singlechirality macroscopic ensembles of single-wall carbon nanotubes (SWCNTs). ${ }^{1-8}$ This impressive progress is revolutionizing the field of carbon nanotube physics and chemistry, enabling detailed chirality-dependent spectroscopy studies that were previously impossible to perform. ${ }^{9-14}$ While many of the features in prior studies were obscured due to the many different chiralities present in the samples, in highly enriched samples these features are better resolved, allowing one to study the intrinsic behaviors of one-dimensional (1D) electrons, phonons, and excitons in SWCNTs in far greater detail. Moreover, the availability of highly purified single-chirality SWCNTs opens up new possibilities for the development of

Received: December 7, 2013

Revised: February 4, 2014

Published: February 14, 2014 
(a)

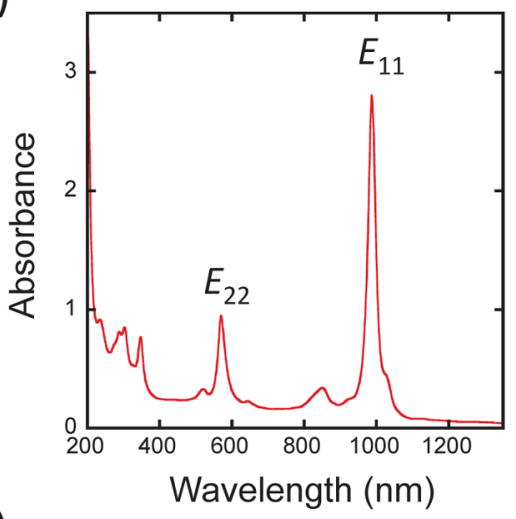

(c)

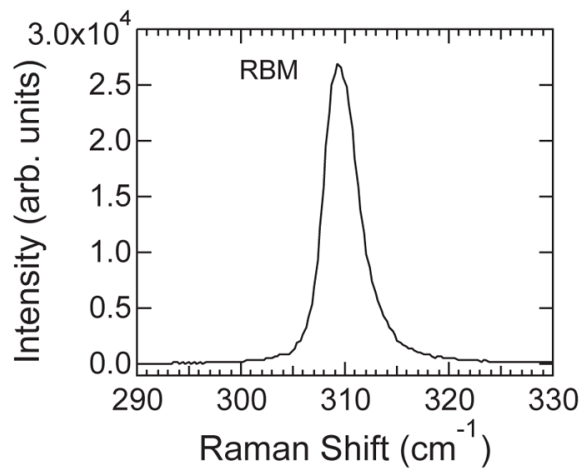

(e)

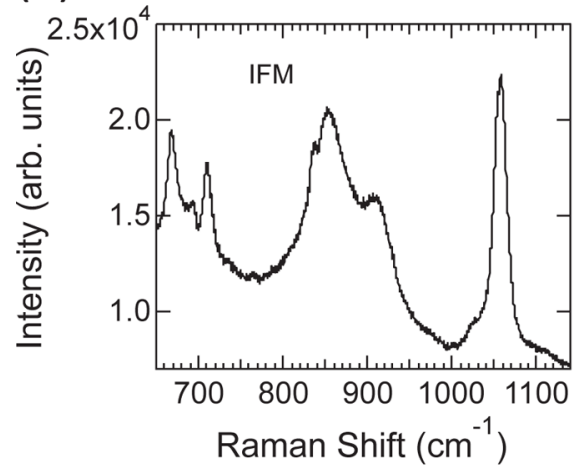

(b)

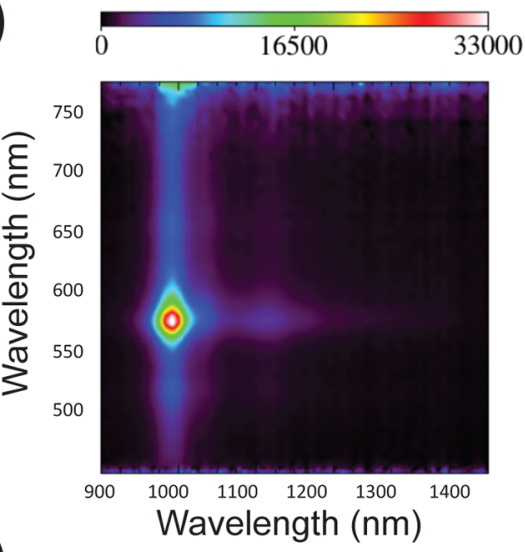

(d)

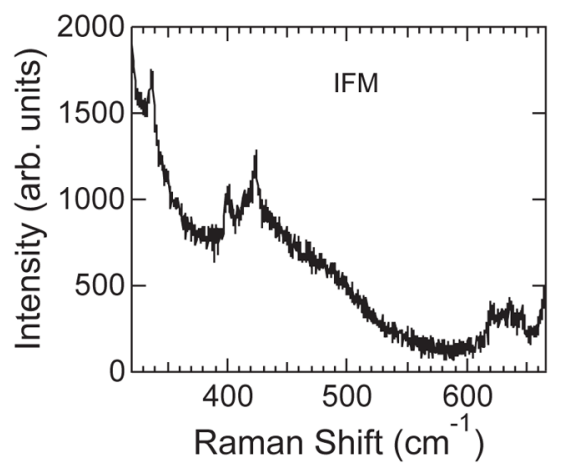

(f)

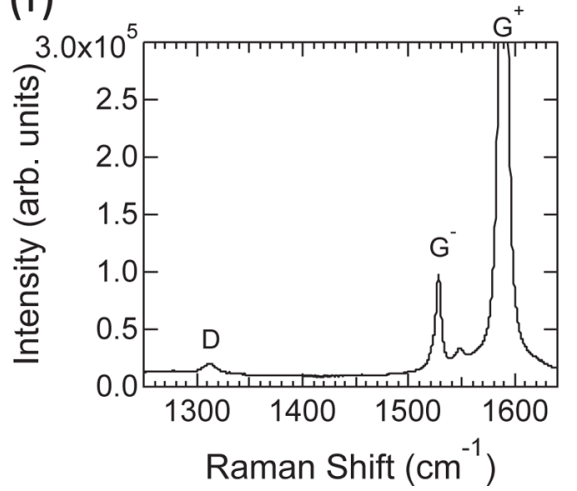

Figure 1. (a) Absorption and (b) photoluminescence excitation spectra for the (6,5)-enriched SWCNT sample. (c-f) Continuous-wave Raman spectra taken with an excitation wavelength of $561.3 \mathrm{~nm}$ for different frequency ranges from 290 to $1640 \mathrm{~cm}^{-1}$. Although the vertical scales are given in arbitrary units, the relative scales are the same for $(c-f)$.

optoelectronic devices $^{15-19}$ that are useful for optical communications, spectroscopy, imaging, and sensing.

In this paper, we focus on the properties of phonons in SWCNTs generated through ultrafast optical excitation on a macroscopic ensemble of highly enriched $(6,5)$ SWCNTs. ${ }^{6}$ Raman spectroscopy has been an indispensable tool for characterizing and understanding the electronic and vibrational properties of graphite, SWCNTs, and graphene. ${ }^{20-25}$ Much accumulated knowledge exists on the strong Raman-active fundamental modes in SWCNTs such as the radial breathing mode (RBM), the D-mode, the G-mode, and the $\mathrm{G}^{\prime}$ (or 2D) mode, observed through resonance Raman scattering spectroscopy. However, in contrast to graphite and graphene, SWCNTs are expected to exhibit many other fundamental modes arising from the large number of $1 \mathrm{D}$ phonon branches that arise from circumferential quantization (or zone folding). At present, there is little experimental information available on these additional modes and their combinational modes, except some Raman studies of intermediate frequency modes (IFMs) in mixedchirality samples. ${ }^{26-30}$ In addition to the problem that they are obscured in chirality-mixed samples, they are also difficult to detect because they couple weakly to the photoexcited carriers.

We utilized some of the advantages of coherent phonon (CP) spectroscopy $^{31}$ to successfully observe and identify 14 distinct phonon features in $(6,5)$ SWCNTs. The two most important advantages of CP spectroscopy for the present study are its excellent frequency resolution $\left(\sim 0.4 \mathrm{~cm}^{-1}\right.$ in the current case) and the ease with which low-frequency modes can be observed due to the absence of the Rayleigh scattering or photoluminescence background. We have observed symmetryallowed acoustic modes (with $\mathrm{E}_{1}$ and $\mathrm{E}_{2}$ symmetries) that are consistent with our calculations based on the extended tight- 
Table 1. Experimentally Measured and Theoretically Predicted Frequencies of Various Coherent Phonon (CP) Modes for (6,5) Single-Wall Carbon Nanotubes, Including Both Fundamental and Combinational Modes, Together with Assignments and CW Raman values ${ }^{a}$

\begin{tabular}{|c|c|c|c|c|}
\hline peak number & $\mathrm{CP}\left(\mathrm{cm}^{-1}\right)$ & assignment & $\operatorname{Raman}\left(\mathrm{cm}^{-1}\right)$ & theory $\left(\mathrm{cm}^{-1}\right.$ \\
\hline \multirow[t]{2}{*}{$\# 1$} & $86 \pm 3$ & 1st $\mathrm{E}_{2}$ (iTA) & & 76 \\
\hline & & 2nd $E_{1}(\mathrm{LA})$ & & 213 \\
\hline \multirow[t]{4}{*}{ \#2 } & $309 \pm 2$ & 3rd A (RBM) & 309 & 294 \\
\hline & & 2nd $E_{2}$ (LA-like) & & 397 \\
\hline & & $3 r d E_{1}$ & 399 & 407 \\
\hline & & 3rd $\mathrm{E}_{1}$ & 423 & 407 \\
\hline \#3 & $487 \pm 4$ & $\mathrm{RBM}+2 \mathrm{nd} \mathrm{E}_{1}$ & & 507 \\
\hline$\# 4$ & $574 \pm 4$ & 1st $E_{2}+2 n d E_{1}+R B M$ & & 582 \\
\hline \multirow[t]{5}{*}{ \#5 } & $623 \pm 9$ & RBM overtone & 621 & 588 \\
\hline & & $\left(\right.$ or oTO -1 st $E_{2}-2$ nd $\left.E_{1}\right)$ & & $(595)$ \\
\hline & & $3 r d E_{2}$ & & 616 \\
\hline & & & 668 & \\
\hline & & & 692 & \\
\hline \#6 & $718 \pm 1$ & $\mathrm{oTO}-2 \mathrm{nd}_{1}$ & 710 & 671 \\
\hline \multirow[t]{7}{*}{$\# 7$} & $807 \pm 5$ & $\mathrm{oTO}-1$ st $\mathrm{E}_{2}$ & & 807 \\
\hline & & & 837 & \\
\hline & & & 853 & \\
\hline & $883 \pm 12$ & 4th A (oTO) & & 884 \\
\hline & & 4th $E_{2}$ & & 874 \\
\hline & & 4th $\mathrm{E}_{1}$ & & 881 \\
\hline & & & 908 & \\
\hline \multirow[t]{2}{*}{$\# 8$} & $970 \pm 6$ & $\mathrm{oTO}+1$ st $\mathrm{E}_{2}$ & & 960 \\
\hline & & & 1027 & \\
\hline \#9 & $1054 \pm 3$ & $\mathrm{oTO}+2 \mathrm{nd} \mathrm{E}_{1}$ & 1057 & 1096 \\
\hline \multirow[t]{5}{*}{$\# 10$} & $1145 \pm 6$ & $\mathrm{oTO}+1 \mathrm{st} \mathrm{E}_{2}+2 \mathrm{nd} \mathrm{E}_{1}$ & & 1173 \\
\hline & & $\mathrm{D}$ & 1312 & 1338 \\
\hline & & 5th $\mathrm{E}_{2}$ & & 1521 \\
\hline & & 5th $\mathrm{E}_{1}$ & & 1568 \\
\hline & & 5th A (iTO or $\mathrm{G}^{-}$) & 1528 & 1575 \\
\hline \multirow[t]{3}{*}{$\# 11$} & $1588 \pm 4$ & 6th $\mathrm{A}\left(\mathrm{LO}\right.$ or $\left.\mathrm{G}^{+}\right)$ & 1589 & 1588 \\
\hline & & 6th $\mathrm{E}_{1}$ & & 1570 \\
\hline & & 6th $\mathrm{E}_{2}$ & & 1548 \\
\hline$\# 12$ & $1764 \pm 4$ & $\mathrm{LO}+2 \mathrm{nd} \mathrm{E}_{1}$ & & 1801 \\
\hline$\# 13$ & $1866 \pm 4$ & $\mathrm{LO}+1$ st $\mathrm{E}_{2}+2$ nd $\mathrm{E}_{1}$ & & 1877 \\
\hline$\# 14$ & $2591 \pm 4$ & $2 \mathrm{D}\left(\right.$ or $\left.\mathrm{G}^{\prime}\right)$ & & 2618 \\
\hline
\end{tabular}

${ }^{a}$ Peak number refers to the labels indicated for the observed CP peaks in Figure $2 \mathrm{c}$. The values in the theory column were calculated through the extended tight-binding model. ${ }^{32}$

binding model. We also observed strong IFMs, which can be assigned to combinations between out-of-plane tangential optical (oTO) and acoustic phonons $\left(\mathrm{E}_{1}, \mathrm{E}_{2}\right.$, and $\mathrm{E}_{1}+\mathrm{E}_{2}$ symmetries). Furthermore, we observed combinational modes between A symmetry optical phonons, that is, the RBM and the LO mode, and acoustic phonons $\left(\mathrm{E}_{2}\right.$ and $\mathrm{E}_{1}+\mathrm{E}_{2}$ symmetries). Finally, we provide a comprehensive list of phonon frequencies for $(6,5)$ SWCNTs with assignments for both fundamental and combinational modes, based on a detailed comparison with our theoretical calculations of the full phonon dispersion curves for the $(6,5)$ SWCNTs.

Figure 1a shows the absorption spectrum in the nearinfrared, visible, and ultraviolet for the highly enriched $(6,5)$ SWCNT sample. The largest absorption peaks are attributed to the lowest-energy interband resonances, $\mathrm{E}_{11}$ and $\mathrm{E}_{22}$, which occur at $985 \mathrm{~nm}(1.26 \mathrm{eV})$ and $575 \mathrm{~nm}(2.16 \mathrm{eV})$, respectively. These values are in good agreement with those reported for other studies of $(6,5)$ SWCNTs. ${ }^{19}$ The other smaller features are due to the phonon sidebands of the $E_{11}$ and $E_{22}$ peaks as well as higher-order $\left(\mathrm{E}_{33}, \mathrm{E}_{44}\right.$, etc. $)$ interband transitions in the shortest wavelength region of the spectrum. The narrow widths of the absorption lines attest to the high quality and purity of the $(6,5)$ samples. Figure $1 \mathrm{~b}$ shows a photoluminescence excitation map for the sample, plotting the excitation wavelength on the vertical axis versus emission wavelength on the horizontal axis. The strong spot observed at an excitation wavelength of $575 \mathrm{~nm}$ and an emission wavelength of $985 \mathrm{~nm}$ is consistent with the $\mathrm{E}_{11}$ and $\mathrm{E}_{22}$ peaks in the absorption spectrum. No other spot is visible in the map, demonstrating the highly chirality-enriched nature of the sample. Figure $1 \mathrm{c}-\mathrm{f}$ shows continuous-wave (CW) Raman scattering spectra, taken via $E_{22}$ resonance at an excitation wavelength of $561.3 \mathrm{~nm}$, for different frequency ranges between 290 and $1640 \mathrm{~cm}^{-1}$. Although the vertical scales are given in arbitrary units, the relative scales are the same for Figure $1 \mathrm{c}-\mathrm{f}$. In addition to the strong RBM (in c) and the D-mode and G-modes (in f), some weaker-intensity IFMs are observed (in $d$ and e). The frequencies of these features are summarized in the fourth column of Table 1 . 

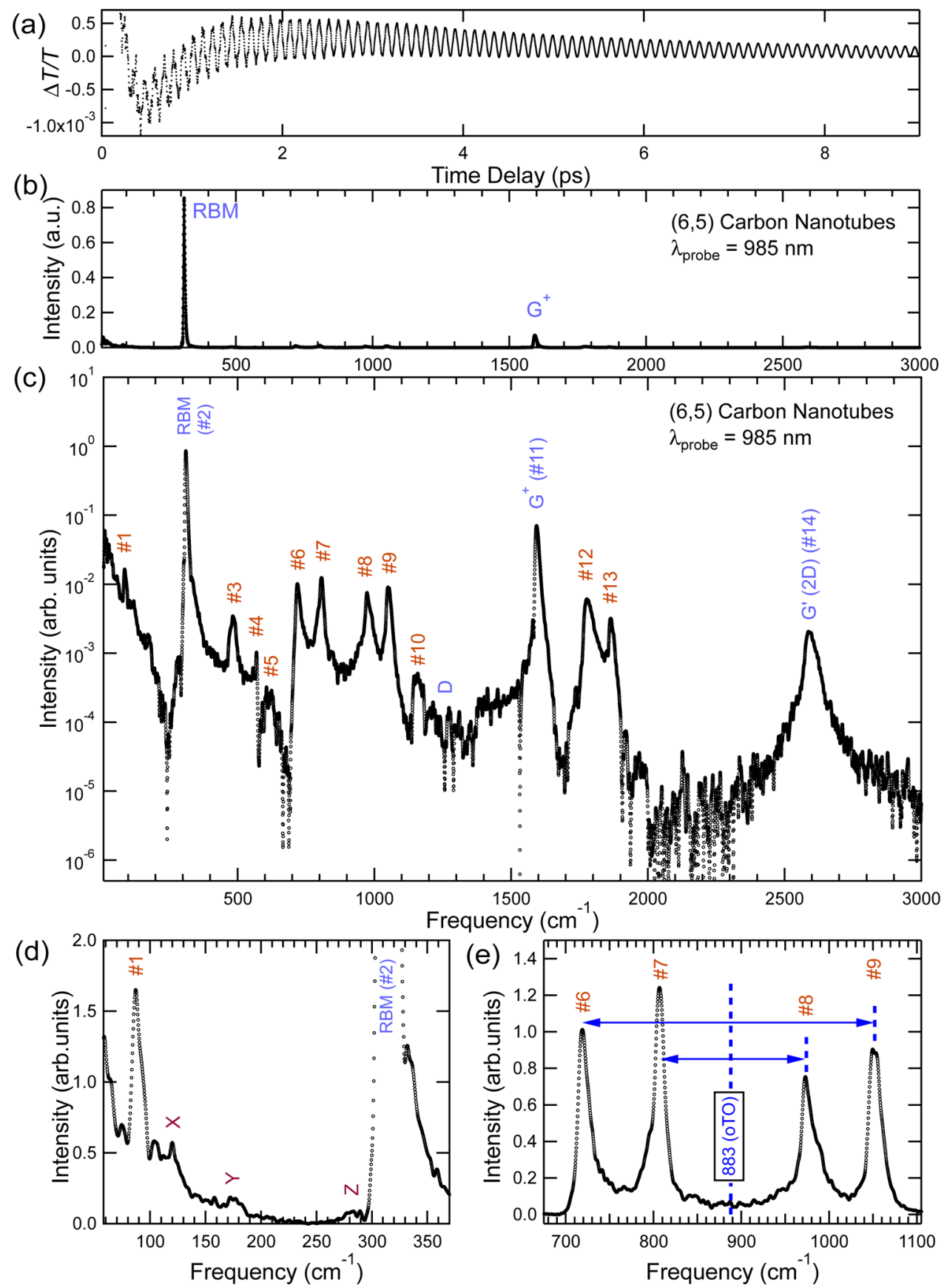

Figure 2. (a) Differential transmission trace at a probe wavelength of $975 \mathrm{~nm}$ for $(6,5)$ single-wall carbon nanotubes showing ultrafast oscillations due to coherent phonons. $(b, c)$ Coherent phonon spectrum obtained through Fourier transformation of the data in (a) in linear and log scales, respectively, showing many phonon modes, labeled \#1-15. (d) The lowest frequency range, showing Peaks \#1-3, together with three less distinct peaks $(\mathrm{X}, \mathrm{Y}$, and $\mathrm{Z})$. (e) Intermediate frequency modes, displaying symmetric pairs as sidebands of the oTO mode $\left(\sim 883 \mathrm{~cm}^{-1}\right)$.

Figure 2a shows a typical differential transmission trace in the time domain taken with $\sim 8$ fs pump pulses with a bandwidth over $400 \mathrm{~nm}$ centered around $800 \mathrm{~nm}$ together with a probe beam with a $5 \mathrm{~nm}$ bandwidth centered at $975 \mathrm{~nm}$. The intensity ratio between the pump and probe beams was $\sim 7 / 2$. The data exhibits ultrafast oscillations, containing various frequency components, the strongest of which are the RBM at $\sim 310$ $\mathrm{cm}^{-1}$ and the $\mathrm{G}^{+}$(LO) mode at $\sim 1590 \mathrm{~cm}^{-1}$, as seen in the Fourier spectrum (Figure $2 b$ ). Figure $2 c$ shows the same spectrum as Figure $2 \mathrm{~b}$ but on a logarithmic scale in order to highlight a number of smaller intensity modes. We can clearly identify 14 distinct features, labeled \#1-14 in Figure 2c, the highest-frequency mode being the $\mathrm{G}^{\prime}$ (or 2D) mode at $\sim 2590$ $\mathrm{cm}^{-1}$. An expanded view of the lowest-frequency region of the spectrum is given in Figure 2d, showing Peak \#1 at $\sim 85 \mathrm{~cm}^{-1}$ and Peak \#2 (RBM) at $\sim 310 \mathrm{~cm}^{-1}$. In addition, there are three less distinct features at $\sim 120, \sim 180$, and $\sim 280 \mathrm{~cm}^{-1}$, which we label "X", "Y", and "Z", respectively. Finally, the IFMs are shown in Figure 2e, displaying the pairwise appearance of four peaks above and below a central frequency of $\sim 883 \mathrm{~cm}^{-1}$. The frequencies of the observed 14 peaks with estimated error bars are listed in the second column of Table 1, together with their respective assignments in the third column, as discussed below. 
In a SWCNT, vibrational modes with $A, E_{1}$, and $E_{2}$ symmetries are Raman active, and there are six modes in each symmetry. ${ }^{20-22,24}$ Among the phonon modes of the same order, the cutting line of the A mode is at the zone center of the graphene $k$-space while the cutting line of the $\mathrm{E}_{1}\left(\mathrm{E}_{2}\right)$ mode is the nearest (next nearest) to the zone center. Among the six A symmetry modes, the RBM, iTO, and LO are expected to have nonzero electron-phonon matrix elements at the $\Gamma$ point $(q=$ $0)$, and two acoustic phonon modes, the "twiston" mode (TW) and the LA mode, are expected to have matrix elements at nonzero $q$. The $\mathrm{E}_{1}$ and $\mathrm{E}_{2}$ symmetry modes have weaker electron-phonon coupling than the A symmetry mode. ${ }^{32}$ Figure 3 a shows the phonon dispersion curves and the density of states for the $(6,5)$ SWCNT calculated using the extended tight-binding model. ${ }^{32}$ It shows all possible $\mathrm{E}_{1}, \mathrm{E}_{2}$, and $\mathrm{E}_{n}$ symmetries as well as A symmetry phonon branches. To investigate possible acoustic phonon modes at frequencies lower than the RBM we enlarge the low-frequency portion of the dispersion curve in Figure $3 \mathrm{~b}$ to display two branches for $\mathrm{A}$
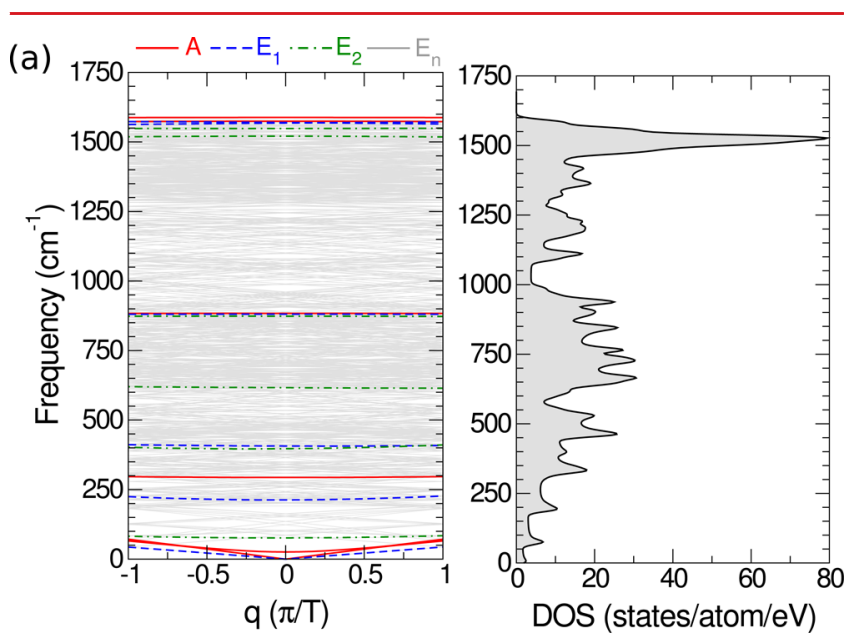

(b)

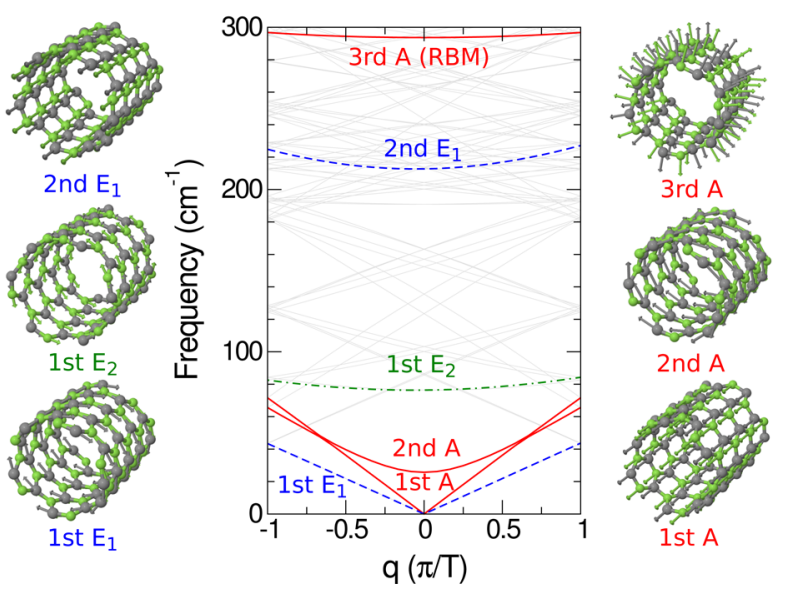

Figure 3. (a) Calculated phonon dispersion curves (left) and corresponding density of states (right) for possible phonon modes $\left(A, E_{1}, E_{2}\right)$ for the $(6,5)$ carbon nanotube, obtained by the extended tight-binding model. Each symmetry mode is distinct from others by its color and has all the same 6-fold phonon branches from the bottom. (b) Enlarged dispersion curve in the low-frequency region with pictures to display the corresponding eigenvector motions of carbon atoms. Note that the 2nd A subband (TW mode), although it is an acoustic mode, has a finite frequency at the $\Gamma$ point due to the use of fitted graphene force constants from the experiment in Ref 38. See also the Methods section. and $E_{1}$ symmetry modes and one branch for the $E_{2}$ symmetry mode. In order to provide more insight into each mode, phonon dynamical matrix eigenvectors are shown in Figure $3 \mathrm{~b}$, whose directions identify these modes as iTA-like or LA-like oscillations. In the last column of Table 1 , we list the $q=0$ frequencies of these fundamental modes and some combinational modes between them. Although curvature effects for the force constants are taken into account in our model, the approximation begins to break down for nanotube diameters smaller than $\sim 0.8 \mathrm{~nm}$ because the long-range force constants are significantly affected by the curvature of the nanotube. For lower-frequency modes, the influence of the curvature effects is relatively small.

On the basis of the frequencies of the calculated phonon modes in Figure 3, we can now discuss the origins of the observed fourteen peaks. First, we attribute Peak \#1 at $\sim 85$ $\mathrm{cm}^{-1}$ to the $\mathrm{E}_{2}$ (iTA-like) mode, whose predicted value is 76 $\mathrm{cm}^{-1}$. Given the uncertainty of $\pm 50 \mathrm{~cm}^{-1}$ in the theoretically calculated values, which depends on the choice of force constants, ${ }^{33}$ this is reasonable agreement. We also speculatively assign the weak and broader peak at $\sim 175 \mathrm{~cm}^{-1}$ (Peak Y) to the $\mathrm{E}_{1}$ (LA-like) mode, whose calculated value is $213 \mathrm{~cm}^{-1}$. In addition, the weak peak near $280 \mathrm{~cm}^{-1}$ (Peak Z), just below the RBM, could be due to a combinational mode between the first $\mathrm{E}_{2}$ (iTA-like, $76 \mathrm{~cm}^{-1}$ ) and the second $\mathrm{E}_{1}$ (LA-like, $213 \mathrm{~cm}^{-1}$ ) modes; such a combinational mode between acoustic phonons has been seen in a graphite whisker ${ }^{34}$ and multiwall carbon nanotubes ${ }^{35}$ and is believed to arise through a doubleresonance process. ${ }^{36}$

As seen in Figure 2e, four pronounced IFM peaks (Peaks \#6-9) appear in pairs, symmetrically separated from the center frequency at $883 \mathrm{~cm}^{-1}$. We interpret this central frequency as that of the oTO mode, which is predicted to have a frequency of $884 \mathrm{~cm}^{-1}$, again in excellent agreement. Then the two pairs of IFM peaks can be understood as the sum $(+)$ and difference (-) frequencies, respectively, of the oTO mode and (i) the first $\mathrm{E}_{2}$ mode (iTA-like, $76 \mathrm{~cm}^{-1}$ ) and (ii) the second $\mathrm{E}_{1}$ mode (LAlike, $\left.213 \mathrm{~cm}^{-1}\right)$. In addition, the weaker IFM peaks at $623 \mathrm{~cm}^{-1}$ (Peak \#5) and $1145 \mathrm{~cm}^{-1}$ (Peak \#10) can also be the sum and difference, respectively, of the oTO mode and the sum of (i) and (ii) (three phonon combinations) although it is also likely that Peak \#5 is the RBM overtone as previously observed in Raman. ${ }^{30}$ Furthermore, there are two other IFM peaks, that is, Peaks \#3 and \#4. Peak \#3 at $487 \mathrm{~cm}^{-1}$ can be assigned to a combinational mode between the RBM $\left(294 \mathrm{~cm}^{-1}\right)$ and the second $\mathrm{E}_{1}$ mode (LA-like, $213 \mathrm{~cm}^{-1}$ ). Peak \#4 at $574 \mathrm{~cm}^{-1}$ can be assigned to a combination of three different symmetry modes, that is, the RBM $\left(A_{1}, 294 \mathrm{~cm}^{-1}\right)$, the second $E_{1}$ mode (LA-like, $213 \mathrm{~cm}^{-1}$ ), and the first $\mathrm{E}_{2}$ mode (iTA-like, $76 \mathrm{~cm}^{-1}$ ).

Previous Raman measurements on chirality-mixed SWCNT samples detected some IFMs in the $600-1100 \mathrm{~cm}^{-1}$ frequency range excited in the $E_{33} / E_{44}$ region $^{26}$ as well as in the $370-480$ $\mathrm{cm}^{-1}$ frequency range excited in the $\mathrm{E}_{11} / \mathrm{E}_{22}$ region. ${ }^{29,30}$ Fantini et al. observed only two peaks associated with the oTO mode combined with acoustic phonon modes. ${ }^{26}$ The so-called "steplike" dispersive behaviors were observed in these studies because the IFMs have a strong chirality- and diameterdependence, which jumps when the excitation photon energy goes from one $2 n+m$ family to another. Kilina and coworkers ${ }^{37}$ suggested some connection between the resonances for these particular IFMs and cross-polarized transitions, which may be reasonable with the E-symmetry character of these modes. However, in all these previous studies on chirality- 
mixed ensembles, the analysis and ultimate $(n, m)$ assignment of specific IFMs were model-dependent, and thus, the determined IFM frequencies depended on the assumption that the $(n, m)$ assignments were correct. On the contrary, the present work on IFM coherent phonons represents a single $(n, m)$ probe of IFM behavior, providing model-independent frequencies.

Lastly, it is interesting to note some distinct differences between Raman and CP spectra. First, Raman observed a clear D-peak at $\sim 1312 \mathrm{~cm}^{-1}$ (see Figure 1f) whereas it was absent in the CP spectra (see Figure 2c). Second, a clear $\mathrm{G}^{-}$peak was observed at $\sim 1528 \mathrm{~cm}^{-1}$ in Raman (see Figure 1f) whereas it was not clearly observed in CP (see Figure 2c). We are currently modeling the $\mathrm{CP}$ generation and detection processes for various phonon modes, including the $\mathrm{D}$ and $\mathrm{G}^{-}$modes, to compare their expected intensities with the experimental observations. Furthermore, in Raman we see an IFM at $\sim 399$, $\sim 420, \sim 668, \sim 692, \sim 837, \sim 853, \sim 908$, and $\sim 1027 \mathrm{~cm}^{-1}$ (see Figure 1d,e and Table 1), which do not agree with any of the clearly observed CP peaks. It should be noted that any short-lived phonon mode cannot be detected in CP spectroscopy because they are significantly affected by the fast and strong electronic pump-probe signal (especially important in SWCNTs). In our CP analysis, we did not use any data before $\sim 125 \mathrm{fs}$, and therefore, if the peak width exceeds $\sim 80 \mathrm{~cm}^{-1}$, the peak is not recorded. This is a possible explanation as to why $\mathrm{CP}$ does not show the broad peaks at 853 and $908 \mathrm{~cm}^{-1}$ observed in Raman. In addition, the phonon generation mechanism by photoexcited carriers (exciton-phonon interaction in the case of SWCNTs) should be common for Raman spectroscopy and ultrafast pump-probe spectroscopy. However, in the case of Raman spectroscopy, we measure the photon energy of the scattered light while in the case of pumpprobe spectroscopy we measure the reflectance or transmittance of the probe beam. The optical response of the material to the probe beam in the presence of the phonons may not always be the same as that for the Raman spectra. The modifications of the probe reflectance and transmittance should depend on phonon vibration amplitudes and directions. A further investigation is under way.

In conclusion, by combining the excellent signal-to-noise ratio and spectral resolution of coherent phonon spectroscopy with a highly enriched, single-chirality $(6,5)$ single-wall carbon nanotube sample, we have detected previously unobserved vibrational modes. The combination of a modern SWCNT separation technique with advanced ultrafast optical spectroscopy allowed direct observations of several phonon branches of $A, E_{1}$, and $E_{2}$ symmetries and their combinations. We demonstrated that there is strong coupling between different phonon modes over a wide frequency range. By presenting a detailed compendium of phonons for single-chirality single-wall carbon nanotubes, this work serves as a reference with which to refine our understanding of the dynamics and interactions of one-dimensional phonons and electrons and as a testbed for new theoretical models for more precise and accurate calculations. Furthermore, the present analysis shows that there is a clear discrepancy for some phonon modes between Raman and pump probe measurements, whose reasons remain to be explained.

Methods. Single-chirality (6,5) SWCNTs with $4 \mathrm{mM}$ concentration were micelle suspended by $0.5 \mathrm{wt} \%$ DOC through the multicolumn gel chromatography isolation method. ${ }^{6}$ Pump-probe differential transmission measurements were performed on the solution in a quartz cell with an optical path length of $1 \mathrm{~mm}$ at room temperature. The light source for $\mathrm{E}_{11}$ excitation was a home-built Ti:Sapphire laser equipped with a double-chirped mirror set (DCM 7, Venteon, Inc.), which delivers pulses as short as 8 fs with a bandwidth over $400 \mathrm{~nm}$ centered around $800 \mathrm{~nm}$. The probe beam was spectrally resolved by a set of bandpass filters with a bandwidth of $5 \mathrm{~nm}$, prior to the photodetector. The phonon dispersions of the $(6,5)$ SWCNT were obtained within an extended (long-range and symmetry-adapted) tight-binding model employing a specific force constant set. ${ }^{33}$ This approach takes into account the curvature effects of the SWCNT in the calculation of the force constant parameters derived from the graphene force constants. The graphene force constant parameters were calculated by fitting the phonon dispersion from inelastic $\mathrm{X}$ ray scattering experiment. ${ }^{38}$

\section{AUTHOR INFORMATION}

\section{Corresponding Authors}

*E-mail: (J.K.) kono@rice.edu.

*E-mail: (T.J.) thjoo@postech.ac.kr

\section{Present Address}

(J.-H.K.) Sungkyunkwan University, Jangan-gu, Suwon, Republic of Korea.

Notes

The authors declare no competing financial interest.

\section{ACKNOWLEDGMENTS}

This work was supported in part by the National Research Foundation of Korea (NRF) grant funded by the Korea government (MEST) (2013R1A1A2006659, 2010-022691). T.J. acknowledges the support by the National Research Foundation of Korea (NRF) grant funded by the Korea government (MSIP) (2007-0056330) and the Global Research Laboratory Program (2009-00439). Y.L. thanks Professor F. X. Kärtner and Dr. H. Kyunghan at MIT for technical support for generating sub-8 fs pulses. J.K. acknowledges support by the Department of Energy (through Grant DE-FG02-06ER46308), the National Science Foundation (through Grant OISE0968405), and the Robert A. Welch Foundation (through Grant C-1509). G.D.S. and C.J.S. acknowledge support from the National Science Foundation through Grants OISE0968405 and DMR-1105437. H.L. acknowledges support by the "100 talents project" of CAS and the recruitment program of global youth experts. H.K. acknowledges support by JSPS KAKENHI Grant 25220602. R.S. acknowledges support by KAKENHI (Nos. 25286005 and 25107005). A.R.T.N. acknowledges the support by the JSPS Research Fellowship for Young Scientists (201303921). Raman spectra were acquired at the Center for Integrated Nanotechnology, a U.S. Department of Energy, Office of Basic Energy Sciences user facility. E.H.H., H.T., and S.K.D. acknowledge partial support from the LANL LDRD program.

\section{REFERENCES}

(1) Zheng, M.; Jagota, A.; Strano, M. S.; Santos, A. P.; Barone, P.; Chou, S. G.; Diner, B. A.; Dresselhaus, M. S.; McLean, R. S.; Onoa, G. B.; Samsonidze, G. G.; Semke, E. D.; Usrey, M.; Walls, D. J. Science 2003, 302, 1545-1548.

(2) Zheng, M.; Jagota, A.; Semke, E. D.; Diner, B. A.; McLean, R. S.; Lustig, S. R.; Richardson, R. E.; Tassi, N. G. Nat. Mat. 2003, 2, 338342.

(3) Arnold, M. S.; Stupp, S. I.; Hersam, M. C. Nano Lett. 2005, 5, $713-718$. 
(4) Arnold, M. S.; Green, A. A.; Hulvat, J. F.; Stupp, S. I.; Hersam, M.

C. Nat. Nanotechnol. 2006, 1, 60-65.

(5) Ghosh, S.; Bachilo, S. M.; Weisman, R. B. Nat. Nanotechnol. 2010, $5,443-450$.

(6) Liu, H.; Nishide, D.; Tanaka, T.; Kataura, H. Nat. Commun. 2011, 2, 309.

(7) Tu, X.; Hight Walker, A. R.; Khripin, C. Y.; Zheng, M. J. Am. Chem. Soc. 2011, 133, 12998-13001.

(8) Khripin, C. Y.; Fagan, J. A.; Zheng, M. J. Am. Chem. Soc. 2013, 135, 6822-6825.

(9) Hároz, E. H.; Rice, W. D.; Lu, B. Y.; Ghosh, S.; Hauge, R. H.; Weisman, R. B.; Doorn, S. K.; Kono, J. ACS Nano 2010, 4, 19551962.

(10) Duque, J. G.; Chen, H.; Swan, A. K.; Shreve, A. P.; Kilina, S.; Tretiak, S.; Tu, X.; Zheng, M.; Doorn, S. K. ACS Nano 2011, 5, 52335241.

(11) Hároz, E. H.; Duque, J. G.; Rice, W. D.; Densmore, C. G.; Kono, J.; Doorn, S. K. Phys. Rev. B 2011, 84, 121403(R).

(12) Hároz, E. H.; Duque, J. G.; Lu, B. Y.; Nikolaev, P.; Arepalli, S.; Hauge, R. H.; Doorn, S. K.; Kono, J. J. Am. Chem. Soc. 2012, 134, 4461-4464.

(13) Hároz, E. H.; Duque, J. G.; Tu, X.; Zheng, M.; Walker, A. R. H.; Hauge, R. H.; Doorn, S. K.; Kono, J. Nanoscale 2013, 5, 1411-1439.

(14) Zhang, Q.; Hároz, E. H.; Jin, Z.; Ren, L.; Wang, X.; Arvidson, R. S.; Lüttge, A.; Kono, J. Nano Lett. 2013, 13, 5991-5996.

(15) Carbon Nanotubes: Synthesis, Structure, Properties, and Applications; Dresselhaus, M. S., Dresselhaus, G., Avouris, P., Eds.; Topics in Applied Physics 18; Springer: Berlin, 2001.

(16) Avouris, P.; Freitag, M.; Perebeinos, V. Nat. Photonics 2008, 2, $341-350$.

(17) Carbon Nanotubes: Advanced Topics in the Synthesis, Structure, Properties and Applications; Jorio, A., Dresselhaus, G., Dresselhaus, M. S., Eds.; Springer: Berlin, 2008.

(18) Léonard, F. The Physics of Carbon Nanotube Devices; William Andrew: Norwich, NY, 2009.

(19) Nanot, S.; Hároz, E. H.; Kim, J.-H.; Hauge, R. H.; Kono, J. Adv. Mater. 2012, 24, 4977-4994.

(20) Saito, R.; Dresselhaus, G.; Dresselhaus, M. S. Physical Properties of Carbon Nanotubes; Imperial College Press: London, 1998.

(21) Reich, S.; Thomsen, C.; Maultzsch, J. Carbon Nanotubes: Basic Concepts and Physical Properties; Wiley-VCH: Weinheim, 2004.

(22) Dresselhaus, M. S.; Jorio, A.; Hofmann, M.; Dresselhaus, G.; Saito, R. Nano Lett. 2010, 10, 751-758.

(23) Saito, R.; Hofmann, M.; Dresselhaus, G.; Jorio, A.; Dresselhaus, M. S. Adv. Phys. 2011, 60, 413-550.

(24) Jorio, A.; Dresselhaus, M. S.; Saito, R.; Dresselhaus, G. F. Raman Spectroscopy in Graphene Related Systems; Wiley-VCH: New York, 2011.

(25) Ferrari, A. C.; Basko, D. M. Nat. Nanotechnol. 2013, 8, 235-246.

(26) Fantini, C.; Jorio, A.; Souza, M.; Saito, R.; Samsonidze, G. G.; Dresselhaus, M. S.; Pimenta, M. A. Phys. Rev. B 2005, 72, 085446.

(27) Chou, S. G.; Plentz, F.; Jiang, J.; Saito, R.; Nezich, D.; Ribeiro, H. B.; Jorio, A.; Pimenta, M. A.; Samsonidze, G. G.; Santos, A. P.; Zheng, M.; Onoa, G. B.; Semke, E. D.; Dresselhaus, G.; Dresselhaus, M. S. Phys. Rev. Lett. 2005, 94, 127402.

(28) Htoon, H.; O’Connell, M. J.; Doorn, S. K.; Klimov, V. I. Phys. Rev. Lett. 2005, 94, 127403.

(29) Luo, Z.; Papadimitrakopoulos, F.; Doorn, S. K. Phys. Rev. B 2007, 75, 205438.

(30) Luo, Z.; Papadimitrakopoulos, F.; Doorn, S. K. Phys. Rev. B 2008, 77, 035421.

(31) Kim, J.-H.; Nugraha, A. R. T.; Booshehri, L. G.; Hároz, E. H.; Sato, K.; Sanders, G. D.; Yee, K.-J.; Lim, Y.-S.; Stanton, C. J.; Saito, R.; Kono, J. Chem. Phys. 2013, 413, 55-80.

(32) Jiang, J.; Saito, R.; Samsonidze, G. G.; Chou, S. G.; Jorio, A.; Dresselhaus, G.; Dresselhaus, M. S. Phys. Rev. B 2005, 72, 235408.

(33) Li, Z. M.; Popov, V. N.; Tang, Z. K. Solid State Commun. 2004, 130, 657-661.
(34) Tan, P.; Hu, C.; Dong, J.; Shen, W.; Zhang, B. Phys. Rev. B 2001, 64, 214301.

(35) Tan, P.; An, L.; Liu, L.; Guo, Z.; Czerw, R.; Carroll, D. L.; Ajayan, P. M.; Zhang, N.; Guo, H. Phys. Rev. B 2002, 66, 245410.

(36) Saito, R.; Jorio, A.; Souza Filho, A. G.; Dresselhaus, G.; Dresselhaus, M. S.; Pimenta, M. A. Phys. Rev. Lett. 2001, 88, 027401.

(37) Kilina, S.; Tretiak, S.; Doorn, S. K.; Luo, Z.; Papadimitrakopoulos, F.; Piryatinski, A.; Saxena, A.; Bishop, A. R. Proc. Natl. Acad. Sci. U.S.A. 2008, 105, 6797-6802.

(38) Maultzsch, J.; Reich, S.; Thomsen, C.; Requardt, H.; Ordejón, P. Phys. Rev. Lett. 2004, 92, 075501. 